\title{
Dynamic analysis of a prey-predator model with state-dependent control strategy and square root response function
}

\author{
Hongxia Liu ${ }^{1,2^{*}}$ and Huidong Cheng ${ }^{1}$
}

\begin{tabular}{l}
\hline${ }^{\text {CCorrespondence: }}$ \\
sdkdlhx@163.com \\
${ }^{1}$ College of Mathematics and \\
Systems Science, Shandong \\
University of Science and \\
Technology, Qingdao, China \\
${ }^{2}$ State Key Laboratory of Mining \\
Disaster Prevention and Control \\
Co-founded by Shandong Province \\
and the Ministry of Science and \\
Technology, Shandong University of \\
Science and Technology, Qingdao, \\
China
\end{tabular}

"Correspondence: sdkdlhx@163.com

${ }^{1}$ College of Mathematics and Systems Science, Shandong

Tech

${ }^{2}$ State Key Laboratory of Mining Disaster Prevention and Control Co-founded by Shandong Province Technology, Shandong University of China

\section{基 Springer}

\begin{abstract}
In this work, a prey-predator model with square root response function under a state-dependent impulse is proposed. Firstly, according to the differential equation geometry theory and the method of successor function, the existence, uniqueness and attractiveness of the order-1 periodic solution are analyzed. Then the stability of the order-1 periodic solution is discussed by the Poincaré criterion for impulsive differential equations. Finally, we show a specific example and carry out numerical simulations to verify the theoretical results.
\end{abstract}

MSC: $34 C 25 ; 34 D 20 ; 92 B 05 ; 34 A 37$

Keywords: Semi-continuous dynamic systems; Order-1 periodic solution; Successor functions; Attractiveness

\section{Introduction}

The herd behavior, such as of drifting herbivores observed in the savanna, is a scenario in which the predator can only interact with the prey along the outer corridor of the prey herd when the prey are attached by the predator. To see the effect of the herd behavior, Braza et al. [1-5] proposed a square root functional response and in [2], Bachchu et al. considered the following prey-predator system:

$$
\left\{\begin{array}{l}
x^{\prime}(t)=r x(t)\left(1-\frac{x(t)}{K}\right)-\alpha \sqrt{x(t)} y(t), \\
y^{\prime}(t)=y(t)(\alpha \beta \sqrt{x(t)}-d)
\end{array}\right.
$$

where the prey population density and the predator population density at time $t$ are denoted by $x(t)$ and $y(t)$, respectively, $r>0$ denotes the intrinsic birth rate when $y \equiv 0, K>0$ refers to the carrying capacity for the prey, $\alpha>0$ is the predation coefficient, the conversion coefficient is represented by $0<\beta<1$ and $d>0$ is the death rate of the predator. Unlike the discussion of the literature [1] as regards the existence of Hopf bifurcations, Bachchu et al. investigated the nonexistence of periodic orbits, and the existence and uniqueness of limit cycles. Then they found the impact of herd behavior mechanism of prey population to the model system analytically. And they also analyzed how herd behavior of prey controls the dynamics of the model system near origin in an ecologically meaningful way.

(c) The Author(s) 2018. This article is distributed under the terms of the Creative Commons Attribution 4.0 International License (http://creativecommons.org/licenses/by/4.0/), which permits unrestricted use, distribution, and reproduction in any medium, provided you give appropriate credit to the original author(s) and the source, provide a link to the Creative Commons license, and indicate if changes were made. 
Herd behavior of the prey makes it difficult for their predators to get food. For a long term in this way, the predator will be endangered, the ecological balance will also be destroyed. To avoid such a disaster, intervention has to be made, such as pest management and harvesting of fish [6-15]. Such interventions can be mathematically modeled by impulsive systems. Based on (1), Sun et al. [16] introduced the state-dependent impulse strategy: when the density of the herbivores reaches the given threshold $h>0$, we will release predators and harvest some herbivores, resulting in

$$
\left\{\begin{array}{l}
x^{\prime}(t)=r x(t)\left(1-\frac{x(t)}{K}\right)-\alpha \sqrt{x(t)} y(t), \\
y^{\prime}(t)=y(t)(\alpha \beta \sqrt{x(t)}-d), \\
\Delta x(t)=-a x(t), \\
\Delta y(t)=b y(t)+c,
\end{array}\right\} \quad x=h,
$$

where $a \in(0,1), b \in(-1, \infty)$, and $c \geq 0$. When $x=h$, the control strategy is taken, and $x, y$ abruptly turn to $(1-a) h$ and $(1+b) y+c$, respectively. However, authors of [16] only investigated the existence of order-1 periodic solution due to the limitations of the method they used. In this study, we will apply the method of geometric analysis for impulsive systems to investigate the existence and uniqueness of order-1 periodic solutions, which has not been done in [16].

The rest of the paper is organized as follows. Section 2 provides some basic definitions and lemmas as preparation. In Section 3, according to the differential equation geometry theory and the method of successor function, we analyze the existence and attractiveness of the order-1 periodic solution of system (2). In addition, sufficient conditions of the stability of the order- 1 periodic solution is obtained by analogy of the Poincaré criterion. In Section 4, we show a specific example and carry out numerical simulations. Finally, we conclude our work.

\section{Preliminaries}

Some basic definitions and lemmas are provided in this section which are necessary for the following discussion.

Definition 2.1 ([17]) Consider the general differential equations with state-dependent impulse

$$
\left\{\begin{array}{l}
x^{\prime}(t)=P(x, y), \\
y^{\prime}(t)=Q(x, y), \\
\triangle x(t)=U(x, y), \\
\triangle y(t)=V(x, y),
\end{array}\right\} \quad(x, y) \notin M,
$$

where $M$ is called impulsive set, and let $N$ be the corresponding phase set. $M$ and $N$ represent the curve line or straight line in the plane $R_{+}^{2}$. Let the initial point $A \in \Omega=R_{+}^{2} \backslash M$, and the function $I$ is a continuous impulse mapping that satisfies $I: M \rightarrow N$. The dynamic system constituted by the definition of solution of impulsive system (3) is defined as a semi-continuous dynamic system, which is denoted $(\Omega, g, I, M)$. 
Figure 1 The diagram of the successor function

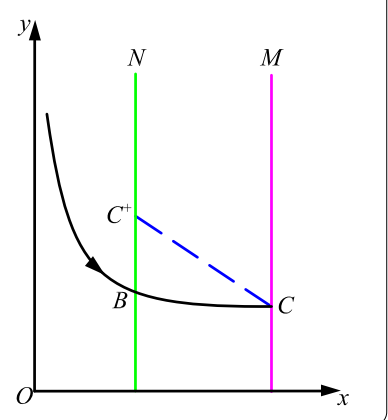

For any $Q \in \Omega$, the map $\Gamma_{Q}: R^{+} \rightarrow \Omega$ defined as $\Gamma_{Q}(t)=\Gamma(Q, t)$ is continuous and we call $\Gamma_{Q}(t)$ the orbit passing through point $Q$. The set $C^{+}(Q)=\{\Gamma(Q, t) \mid 0 \leq t<+\infty\}$ and the set $C^{-}(Q)=\{\Gamma(Q, t) \mid-\infty<t \leq 0\}$ is called positive semi-orbit and the negative semiorbit of point $Q$, respectively. For convenience, if $Q \in \Omega-M, g(Q)$ is called the first point of intersection of $C^{+}(Q)$ and $M$. For any point $B \in \Omega-N$, we define $\Pi(B)$ as the first point of $C^{-}(Q)$ and $N$.

Remark 2.1 Based on system (2), we get $M=\{(x, y) \mid x=h, y \geq 0\}, N=\{(x, y) \mid x=(1-$ $a) h, y \geq 0\}$, for any point $(x, y) \in M$, when $x=h$, we get $I:(h, y) \in M \rightarrow((1-a) h,(1+b) y+$ c) $\in N$.

Definition 2.2 ([18]) Assuming that the impulse set $M$ and the phase set $N$ are both straight lines, as shown in Figure 1 . For any point $B \in N$, then $\Gamma(B, t)=C \in M, I(C)=$ $C^{+} \in N$, we denote the ordinates of point $B$ and $C^{+}$are $y_{B}$ and $y_{B^{+}}$, respectively. Then $C^{+}$is defined as the successor point of $B$, and $f(B)=y_{C^{+}}-y_{B}$ is the successor function of point $B$.

Definition 2.3 ([19]) An orbit $\widetilde{\Gamma}\left(Q_{0}, T\right)$ is called order-1 periodic solution with pe$\operatorname{riod} T$ if there exist a point $Q_{0} \in N$ and $T>0$ such that $Q=\Gamma\left(Q_{0}, T\right) \in M$ and $Q^{+}=$ $I(Q)=Q_{0}$.

Lemma 2.1 ([20]) In system (3), if there exist $A \in N, B \in N$ satisfying the successor function $f(A) f(B)<0$, then there must exist a point $S(S \in N)$ satisfying $S$ between point $A$ and point $B$ such that $f(S)=0$, then system (3) has an order-1 periodic solution.

Lemma 2.2 ([21, 22], Analogue of the Poincaré criterion) The T-periodic solution $(\phi(t), \varphi(t))$ of the system

$$
\left\{\begin{array}{l}
x^{\prime}(t)=P(x, y), \\
y^{\prime}(t)=Q(x, y), \\
\triangle x(t)=\Phi(x, y), \\
\triangle y(t)=\Psi(x, y),
\end{array}\right\} \quad \text { if } \eta(x, y)=0,
$$


has orbital asymptotic stability, if the multiplier $\mu_{2}$ satisfies the condition $\left|\mu_{2}\right|<1$, where

$$
\begin{aligned}
\mu_{2} & =\prod_{i=1}^{q} \Delta_{i} \exp \int_{0}^{T}\left[\frac{\partial P}{\partial x}(\phi(t), \varphi(t))+\frac{\partial Q}{\partial y}(\phi(t), \varphi(t))\right] d t \\
\Delta_{i} & =\frac{P_{+}\left(\frac{\partial \Psi}{\partial y} \frac{\partial \eta}{\partial x}-\frac{\partial \Psi}{\partial x} \frac{\partial \eta}{\partial y}+\frac{\partial \eta}{\partial x}\right)+Q_{+}\left(\frac{\partial \Phi}{\partial x} \frac{\partial \eta}{\partial y}-\frac{\partial \Phi}{\partial y} \frac{\partial \eta}{\partial x}+\frac{\partial \eta}{\partial y}\right)}{P \frac{\partial \eta}{\partial x}+Q \frac{\partial \eta}{\partial y}}
\end{aligned}
$$

and $P, Q, \frac{\partial \Phi}{\partial x}, \frac{\partial \Phi}{\partial y}, \frac{\partial \Psi}{\partial x}, \frac{\partial \Psi}{\partial y}, \frac{\partial \eta}{\partial x}, \frac{\partial \eta}{\partial y}$ are calculated at the point $\left(\phi\left(T_{i}\right), \varphi\left(T_{i}\right)\right)$ and $P_{+}=$ $P\left(\phi\left(T_{i}^{+}\right), \varphi\left(T_{i}^{+}\right)\right), Q_{+}=Q\left(\phi\left(T_{i}^{+}\right), \varphi\left(T_{i}^{+}\right)\right)$.

\section{Dynamical analysis of system (2)}

In this section, the dynamical properties of the order- 1 periodic solution of system (2) are analyzed. Firstly, the qualitative characteristics of system (2) without impulse are discussed. System (2) without impulse is as follows:

$$
\left\{\begin{array}{l}
x^{\prime}(t)=r x(t)\left(1-\frac{x(t)}{K}\right)-\alpha \sqrt{x(t)} y(t)=P(x, y) \\
y^{\prime}(t)=y(t)(\alpha \beta \sqrt{x(t)}-d)=Q(x, y) .
\end{array}\right.
$$

Solving the equations

$$
\left\{\begin{array}{l}
r x(t)\left(1-\frac{x(t)}{K}\right)-\alpha \sqrt{x(t)} y(t)=0 \\
y(t)(\alpha \beta \sqrt{x(t)}-d)=0
\end{array}\right.
$$

yields three equilibria: $O(0,0), A(K, 0), E\left(x_{E}, y_{E}\right)$ of system (4), where $x_{E}=\frac{d^{2}}{\alpha^{2} \beta^{2}}, y_{E}=$ $\frac{r d}{\alpha \beta}\left(1-\frac{d^{2}}{K \alpha^{2} \beta^{2}}\right)$. Let $\left(H_{1}\right): K>\frac{d^{2}}{\alpha^{2} \beta^{2}}$ and $\left(H_{2}\right): 2 r \beta\left(1-\frac{d^{2}}{K \alpha^{2} \beta^{2}}\right)>\left(\frac{r}{2}-\frac{3 r d^{2}}{2 K \alpha^{2} \beta^{2}}\right)^{2}$, then we get the following theorem.

Theorem 3.1 The positive equilibrium $E$ of system (4) is a stable focus if and only if the conditions $\left(H_{1}\right)$ and $\left(H_{2}\right)$ hold.

Proof The Jacobian matrix at equilibrium $E$ is shown as follows:

$$
J(E)=\left(\begin{array}{cc}
\frac{r}{2}-\frac{3 r d^{2}}{2 K \alpha^{2} \beta^{2}} & -\frac{d}{\beta} \\
\frac{r \beta}{2}\left(1-\frac{d^{2}}{K \alpha^{2} \beta^{2}}\right) & 0
\end{array}\right) .
$$

The characteristic equation of $J(E)$ satisfies $f(\lambda)=\lambda^{2}+p \lambda+q=0$, where $p=\frac{r}{2}-\frac{3 r d^{2}}{2 K \alpha^{2} \beta^{2}}$, $q=\frac{r d}{2}\left(1-\frac{d^{2}}{K \alpha^{2} \beta^{2}}\right)$. It is easy to see $\Delta=p^{2}-4 q=\left(\frac{3 r d^{2}}{2 K \alpha^{2} \beta^{2}}-\frac{r}{2}\right)^{2}-2 r d\left(1-\frac{d^{2}}{K \alpha^{2} \beta^{2}}\right)<0$. Then the positive equilibrium $E$ is a stable focus. That completes the proof (see Figure 2).

\subsection{Existence of order-1 periodic solution of system (2)}

On the basis of the ecological significance, system (2) should meet $0<(1-a) h<h<K$. In this paper, the coordinate of arbitrary point $B \in R_{2}^{+}$is denoted $\left(x_{B}, y_{B}\right)$. By Theorem 3.1 , we know the $x$-isoline $L_{1}$ intersects $y$-isoline $L_{2}$ at point $E\left(x_{E}, y_{E}\right)$. For different $h$, we discuss two cases as follows. 
Figure 2 Phase diagram of system (4) with $r=1.2$, $K=1.8, \alpha=0.85, \beta=0.7, d=0.5$

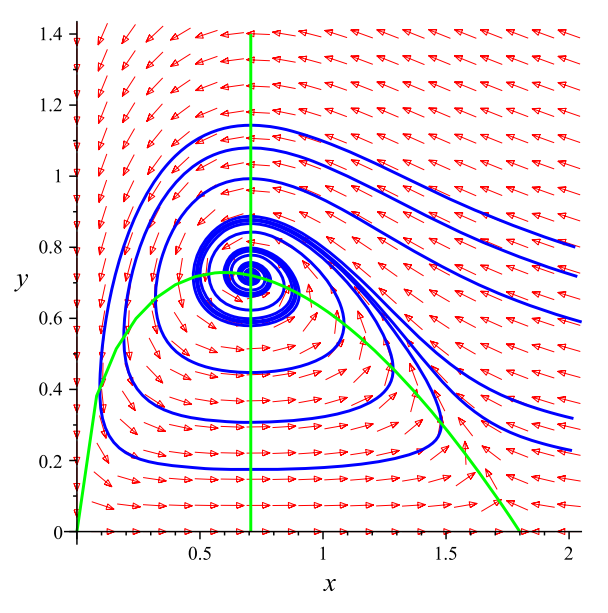

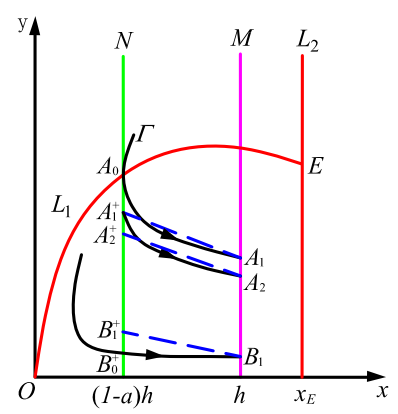

(a)

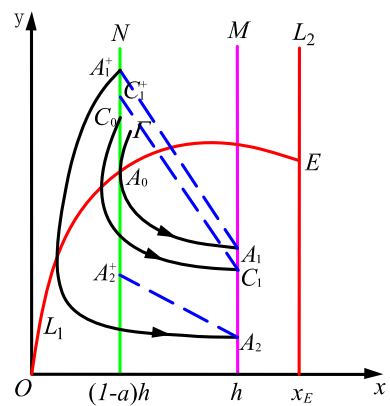

(b)

Figure 3 The schematic of the existence of the order-1 periodic solution in Case I. (a) Discussion in subcase $y_{A_{1}^{+}}<y_{A_{0}}$. (b) Discussion in subcase $y_{A_{1}^{+}}>y_{A_{0}}$

Case I. $0<(1-a) h<h \leq x_{E}<K$.

For notation simplicity, let the intersection of the phase set $N$ and the $x$-isoline $L_{1}$ be $A_{0}\left((1-a) h, y_{A_{0}}\right)$. For system (2), there must exist an orbit $\Gamma$ tangent to set $N$ and intersect with set $M$ at a point $A_{1}\left(h, y_{A_{1}}\right)$, namely, $g\left(A_{0}\right)=A_{1}$, then the point $A_{1}$ jumps to a point $A_{1}^{+}\left((1-a) h, y_{A_{1}^{+}}\right) \in N$ under the action of impulse, where $y_{A_{1}^{+}}=(1+b) y_{A_{1}}+c$. Then the successor function of point $A_{0}$ is $f\left(A_{0}\right)=y_{A_{1}^{+}}-y_{A_{0}}$. Consider the following three subcases based on the different position of point $A_{1}^{+}$.

If $y_{A_{1}^{+}}=y_{A_{0}}$, then $f\left(A_{0}\right)=0$, thus the orbit $\widehat{A_{0} A_{1}}$ and segment $\overline{A_{1} A_{0}}$ constitute an order-1 periodic solution.

If $y_{A_{1}^{+}}<y_{A_{0}}$, then $f\left(A_{0}\right)<0$. Let $g\left(A_{1}^{+}\right)=A_{2} \in M$, under the action of impulse, $A_{2}$ jumps to $A_{2}^{+} \in N$. Because any two orbits are disjoint, then we get $y_{A_{2}}<y_{A_{1}}$ and $y_{A_{2}^{+}}<y_{A_{1}^{+}}$, thus $f\left(A_{1}^{+}\right)=y_{A_{2}^{+}}-y_{A_{1}^{+}}<0$. We can choose another point $B_{0}^{+}\left((1-a) h, y_{B_{0}^{+}}\right) \in N$, where $y_{B_{0}^{+}}=\delta \in$ $(0, c)\left(\delta>0\right.$ is small enough). Let $g\left(B_{0}^{+}\right)=B_{1} \in M$ and $B_{1}$ jumps to $B_{1}^{+} \in N$ under the action of impulse, then $y_{B_{1}^{+}}=(1-a) y_{B_{1}}+c>\delta$. Thus we have $f\left(B_{0}^{+}\right)=y_{B_{1}^{+}}-y_{B_{0}^{+}}>0$. Then there exists a point $B \in N$ satisfying $0<y_{B}<y_{A_{1}^{+}}$such that $f(B)=0$ (see Figure 3(a)).

If $y_{A_{1}^{+}}>y_{A_{0}}$, then $f\left(A_{0}\right)>0$. Let $g\left(A_{1}^{+}\right)=A_{2} \in M$, under the action of impulse, $A_{2}$ jumps to a point $A_{2}^{+} \in N$. Because any two orbits are disjoint, then we get $y_{A_{2}}<y_{A_{1}}$ and $y_{A_{2}^{+}}<y_{A_{1}^{+}}$, thus $f\left(A_{1}^{+}\right)=y_{A_{2}^{+}}-y_{A_{1}^{+}}<0$. We can choose another point $C_{0}\left((1-a) h, y_{A_{0}}+\delta\right) \in N$. Then 


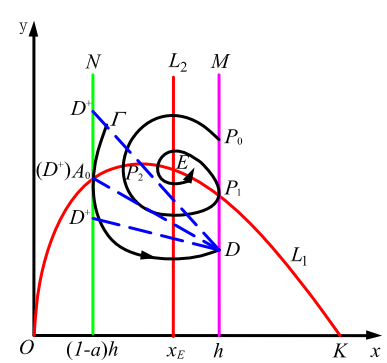

(a)

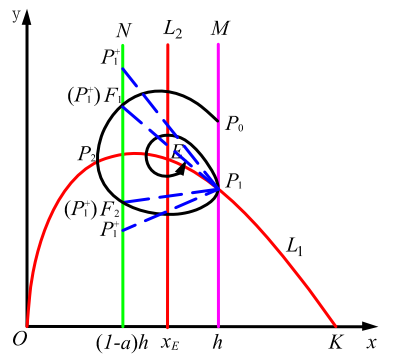

(b)

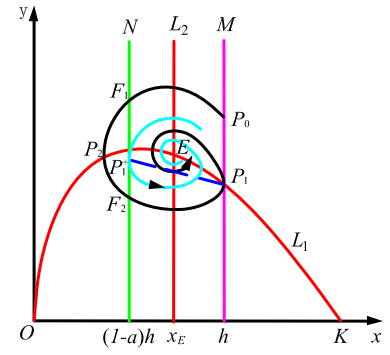

(c)

Figure 4 The schematic of the existence of the order-1 periodic solution in Case II. (a) Discussion in subcase $x_{P_{2}} \geq(1-a) h$. (b) Discussion in subcase $y_{P_{1}^{+}}>y_{F_{1}}$ or $y_{P_{1}^{+}}<y_{F_{2}}$. (c) Discussion in subcase $y_{F_{1}}<y_{P_{1}^{+}}<y_{F_{2}}$

$g\left(C_{0}\right)=C_{1} \in M$ and under the action of impulse, $C_{1}$ jumps to a point $C_{1}^{+} \in N$, then $y_{C_{1}^{+}}=$ $(1+b) y_{C_{1}}+c>\delta$. Due to any two orbits are disjoint, then $y_{C_{1}}<y_{A_{1}}$ and point $C_{1}$ is very close to point $A_{1}$, thus $y_{C_{1}^{+}}<y_{A_{1}^{+}}$and point $C_{1}^{+}$is very close to point $A_{1}^{+}$. Since $y_{A_{1}^{+}}<y_{A_{0}}$, we have $f\left(C_{0}\right)=y_{C_{1}^{+}}-y_{C_{0}}>0$. Therefore, there must be a point $B \in \overline{A_{0} A_{1}^{+}}$such that $f(B)=0$ (see Figure 3(b)).

Case II. $0<(1-a) h<x_{E}<h<K$.

Let the impulsive set $M$ intersect isocline $L_{1}$ at point $P_{1}\left(h, y_{P_{1}}\right)$. The orbit staring from point $P_{1}$ tangents to $M$ at point $P_{1}$, and intersects $L_{1}$ and $M$ at point $P_{2}\left(x_{P_{2}}, y_{P_{2}}\right)$ and $P_{0}\left(h, y_{P_{0}}\right)$, respectively.

If $x_{P_{2}} \geq(1-a) h$, the orbit $\Gamma$ passing through $A_{0}$ tangents to the phase set $N$ at point $A_{0}$, let $g\left(A_{0}\right)=D \in M$, under the action of impulse, point $D$ jumps to point $D_{+} \in N$. According to the discussion of Case I, the order-1 periodic solution of system (2) in this subcase is existent (see Figure 4(a)).

If $x_{P_{2}}<(1-a) h$, the orbit passing through $P_{1}$ intersects set $N$ at points $F_{1}\left((1-a) h, y_{F_{1}}\right)$ and $F_{2}\left((1-a) h, y_{F_{2}}\right)$. Assume $P_{1}$ jumps to $P_{1}^{+}\left((1-a) h, y_{P_{1}^{+}}\right) \in N$ under the action of impulse, there are three subcases to be discussed.

If $y_{P_{1}^{+}}=y_{F_{1}}$ or $y_{P_{1}^{+}}=y_{F_{2}}$, then the segment $\overline{P_{1} F_{1}}$ and the orbit $\widehat{F_{1} F_{2} P_{1}}$ or the segment $\overline{P_{1} F_{2}}$ and the orbit $\widehat{F}_{2} P_{1}$ constitute an order-1 periodic solution of system (2).

If $y_{P_{1}^{+}}>y_{F_{1}}$ or $y_{P_{1}^{+}}<y_{F_{2}}$, according to the analysis of Case I, system (2) exists an order-1 periodic solution (see Figure 4(b)).

If $y_{F_{1}}<y_{P_{1}^{+}}<y_{F_{2}}$, due to any two orbits are disjoint, then the orbit passing through point $P_{1}^{+}$tangents to the phase set $N$ at point $P_{1}^{+}$, and does not intersect with the impulsive set $M$, thus the order-1 periodic solution is nonexistent (see Figure $4(\mathrm{c})$ ). The reader can find the detailed proof in reference [23].

We obtain the following theorem by the above discussions.

Theorem 3.2 Suppose the conditions $\left(H_{1}\right)$ and $\left(H_{2}\right)$ hold.

(I) If $0<(1-a) h<h \leq x_{E}<K$, then the order-1 periodic solution is existent in system

(II) If $0<(1-a) h<x_{E}<h<K$, there are the following three subcases:

(i) If $x_{P_{2}} \geq(1-a) h$, the order-1 periodic solution is existent in system (2).

(ii) If $x_{P_{2}}<(1-a) h, y_{P_{1}^{+}} \geq y_{F_{1}}$ or $y_{P_{1}^{+}} \leq y_{F_{2}}$, the order-1 periodic solution is existent in system (2).

(iii) If $x_{P_{2}}<(1-a) h$ and $y_{F_{1}}<y_{P_{1}^{+}}<y_{F_{2}}$, the order-1 periodic solution is nonexistent. 


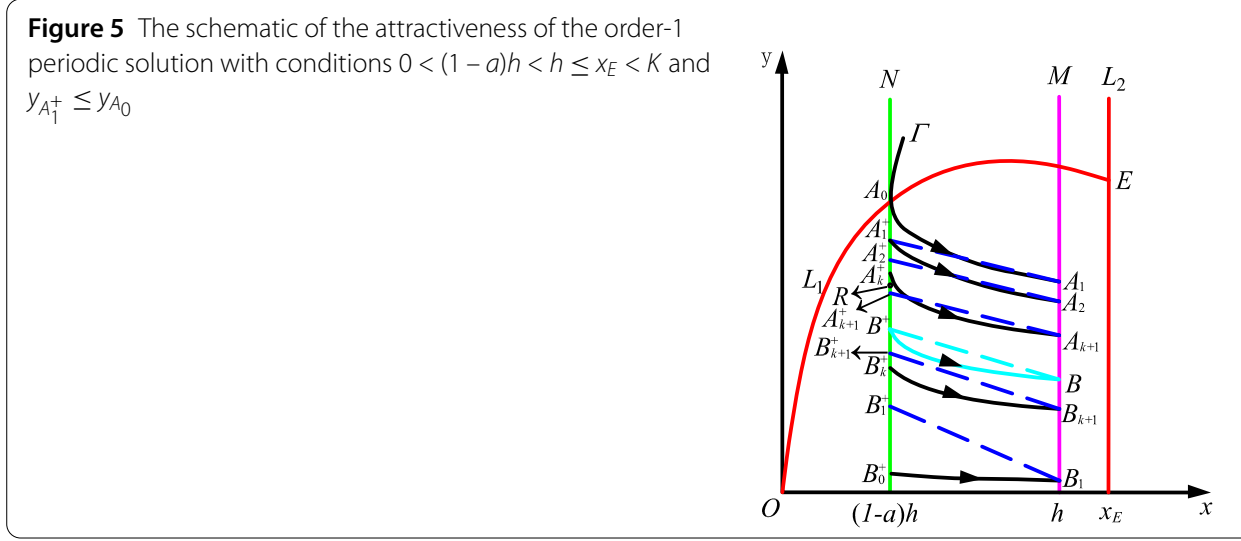

\subsection{Attractiveness of order-1 periodic solution of system (2)}

Based on the conditions of Theorem 3.2, let the initial value $x_{0}<h$, the attractiveness of order-1 periodic solution of system (2) is discussed in this subsection. We mainly discuss Case I, similarly, we can get similar conclusions about Cases II.

Theorem 3.3 If the conditions of $0<(1-a) h<h \leq x_{E}<K$ and $y_{A_{1}^{+}} \leq y_{A_{0}}$ hold, and system (2) exists a unique order-1 periodic solution, then the periodic solution in region $\Omega_{1}=\left\{(x, y) \mid(x, y) \in R_{+}^{2}, x_{0}<h\right\}$ is attractive.

Proof Suppose segment $\overline{B B^{+}}$and orbit $\widehat{B^{+} B}$ constitute the unique order-1 periodic solution (see Figure 5).

Firstly, we choose a point $B_{0}^{+}((1-a) h, \delta) \in N$ which satisfies $\delta<c$ and $y_{B_{0}^{+}}<y_{B^{+}}$. Let $g\left(B_{0}^{+}\right)=B_{1} \in M$, under the action of impulse, $B_{1}$ jumps to $B_{1}^{+} \in N$, then $y_{B_{1}}<y_{B}$, thus we have $y_{B_{1}^{+}}<y_{B^{+}}$. Since $y_{B_{1}^{+}}=(1+b) y_{B_{1}}+c>\delta, f\left(B_{0}^{+}\right)=y_{B_{1}^{+}}-y_{B_{0}^{+}}>0$. Let $g\left(B_{1}^{+}\right)=B_{2} \in M$, then $B_{2}$ jumps to $B_{2}^{+} \in N$. Since $y_{B_{0}^{+}}<y_{B_{1}^{+}}<y_{B^{+}}$, we have $y_{B_{1}}<y_{B_{2}}<y_{B}$, then $y_{B_{1}^{+}}<y_{B_{2}^{+}}<y_{B^{+}}$and $f\left(B_{1}^{+}\right)=y_{B_{2}^{+}}-y_{B_{1}^{+}}>0$. Repeating the process, then we obtain a sequence $\left\{B_{i}^{+}\right\}_{i=0,1,2, \ldots} \in N$ such that $y_{B_{0}^{+}}<y_{B_{1}^{+}}<\cdots<y_{B_{i}^{+}}<\cdots<y_{B^{+}}$and $f\left(B_{i}^{+}\right)=y_{B_{i+1}^{+}}-y_{B_{i}^{+}}>0$. Thus, $\left\{y_{B_{i}^{+}}\right\}_{i=0,1,2, \ldots}$ is monotonically increasing. Hence, $\lim _{i \rightarrow+\infty} y_{B_{i}^{+}}$exists. Next we prove $\lim _{i \rightarrow+\infty} y_{B_{i}^{+}}=y_{B^{+}}$. Assume $B_{*}^{+}=\lim _{i \rightarrow+\infty} B_{i}^{+}$, then we prove $B_{*}^{+}=B^{+}$. Otherwise, $B_{*}^{+} \neq B^{+}$. Let $g\left(B_{*}^{+}\right)=\bar{B} \in M$, then $\bar{B}$ jumps to $\bar{B}^{+} \in N$ by impulsive effect. Then $y_{\bar{B}}<y_{B}, y_{\bar{B}^{+}}<y_{B^{+}}$. Because $f\left(B_{*}^{+}\right) \geq 0$, $B_{*}^{+} \neq B^{+}$, and the periodic solution $\widehat{B^{+} B B^{+}}$is unique, $f\left(B_{*}^{+}\right)=y_{\bar{B}^{+}}-y_{B_{*}^{+}}>0$, thus $y_{B_{*}^{+}}<y_{\bar{B}^{+}}<$ $y_{B^{+}}$. Let $g\left(\bar{B}^{+}\right)=\overline{\bar{B}} \in M$, then $\overline{\bar{B}}$ jumps to $\overline{\bar{B}}^{+}$by impulsive effect. It is easy to know, $y_{\bar{B}}<$ $y_{\bar{B}}<y_{B}$ and $y_{\bar{B}^{+}}<y_{\bar{B}^{+}}<y_{B^{+}}$, then $f\left(\bar{B}^{+}\right)=y_{\bar{B}^{+}}-y_{\bar{B}^{+}}>0$, this is contradictory to the fact that $B_{*}^{+}=\lim _{i \rightarrow+\infty} B_{i}^{+}$, therefore, $B_{*}^{+}=B^{+}$and $\lim _{i \rightarrow+\infty} y_{B_{i}^{+}}=y_{B^{+}}$.

On the other hand, according to the analysis of Case I, we know $y_{B^{+}}<y_{A_{2}^{+}}<y_{A_{1}^{+}}<y_{A_{0}}$, $y_{B}<y_{A_{2}}<y_{A_{1}}$ and $f\left(A_{1}^{+}\right)=y_{A_{2}^{+}}-y_{A_{1}^{+}}<0$. Let $g\left(A_{2}^{+}\right)=A_{3} \in M$, under the action of impulse, $A_{3}$ jumps to $A_{3}^{+} \in N$, then $y_{B^{+}}<y_{A_{3}^{+}}<y_{A_{2}^{+}}, f\left(A_{2}^{+}\right)=y_{A_{3}^{+}}-y_{A_{2}^{+}}<0$. Repeat the process, we obtain a sequence $\left\{A_{k}^{+}\right\}_{k=1,2, \ldots} \in N$ satisfying $y_{A_{1}^{+}}>y_{A_{2}^{+}}>\cdots>y_{A_{k}^{+}}>\cdots>y_{S^{+}}$and $f\left(A_{k}^{+}\right)=y_{A_{k+1}^{+}}-y_{A_{k}^{+}}<0$. Thus, $\left\{y_{A_{k}^{+}}\right\}_{k=1,2, \ldots}$ is monotonically decreasing. Hence, $\lim _{k \rightarrow+\infty} y_{A_{k}^{+}}$ is existent. Similarly, we can prove $\lim _{k \rightarrow+\infty} y_{A_{k}^{+}}=y_{B^{+}}$.

Due to the orbit with arbitrary point of $\Omega_{1}$ will intersect with $N$, next we just prove arbitrary orbit that passes through $N$ eventually is attracted to the periodic solution $\widehat{B^{+} B B^{+}}$.

Choose an arbitrary point $R \in N$ below $A_{0}$ such that $y_{R} \in\left[y_{A_{k+1}^{+}}, y_{A_{k}^{+}}\right)_{k=1,2, \ldots}$. The orbit starting from $R$ moves between orbit $\widehat{A_{k}^{+} A_{k+1}}$ and $\widehat{A_{k+1}^{+} A_{k+2}}$ intersects with $M$ at a point 
in segment $\overline{A_{k+2} A_{k+1}}$, then jumps to a point of $N$ in segment $\overline{A_{k+2}^{+} A_{k+1}^{+}}$, the orbit continues to move between $\widehat{A_{k+1}^{+} A_{k+2}}$ and $\widehat{A_{k+2}^{+} A_{k+3}}$. Repeat the process indefinitely, because $\lim _{k \rightarrow+\infty} y_{A_{k}^{+}}=y_{B^{+}}$, the intersection sequence of orbit which passes through $R$ and the phase set $N$ will be attracted to point $B^{+}$eventually. Similarly, if $y_{R} \in\left[y_{B_{i}^{+}}, y_{B_{i+1}^{+}}\right)_{i=0,1,2, \ldots}$, we also can obtain the intersection sequence of orbit which passes through $R$ and the phase set $N$ will be attracted to point $B^{+}$eventually. Therefore, the orbit starting from arbitrary point below $A_{0}$ eventually is attracted to the periodic solution $\widehat{B^{+} B B^{+}}$.

The orbit with arbitrary point above $A_{0}$ of the phase set $N$ will intersect with $N$ at some point below $A_{0}$ as time goes on, similar to the above discussion, the orbit with arbitrary point above $A_{0}$ will be eventually attracted to the periodic solution $\widehat{B^{+} B B^{+}}$.

Based on the above analysis, the orbit with arbitrary point of $N$ will eventually attracted to periodic solution $\widehat{B^{+} B B^{+}}$. Thus, in the region $\Omega_{1}$, the periodic solution $\widehat{B^{+} B B^{+}}$is attractive. This completes the proof.

See Figure 6(a); let the orbit passing through point $A_{1}^{+}$intersect $N$ at point $G^{+}$and $g\left(G^{+}\right)=G_{1} \in M$, then $G_{1}$ jumps to point $G_{1}^{+} \in N$ under the action of the impulse.

Theorem 3.4 If the conditions of $0<(1-a) h<h \leq x_{E}<K$ and $y_{A_{0}}<y_{G_{1}^{+}}<y_{A_{1}^{+}}$hold, then system (2) exists a unique order-1 periodic solution in region $\Omega_{1}$ which is attractive.

Proof We still suppose that $\widehat{B^{+} B B^{+}}$is the order-1 periodic solution of system (2). Firstly, we analyze the uniqueness of $\widehat{B^{+} B B^{+}}$.

Select two points $I_{0}, J_{0} \in \overline{A_{0} A_{1}^{+}}$such that $y_{J_{0}}>y_{I_{0}}>y_{A_{0}}$, the orbits starting from points $J_{0}$ and $I_{0}$, respectively, intersect $M$ at $J_{1}, I_{1}$, then jump to $J_{1}^{+}, I_{1}^{+} \in N$ under the action of impulse, respectively (see Figure 6(b)). Because any two orbits are disjoint, then $y_{I_{1}}>y_{I_{1}}$, $y_{I_{1}^{+}}>y_{J_{1}^{+}}, f\left(J_{0}\right)=y_{J_{1}^{+}}-y_{J_{0}}, f\left(I_{0}\right)=y_{I_{1}^{+}}-y_{I_{0}}$, we get $f\left(J_{0}\right)-f\left(I_{0}\right)=\left(y_{J_{1}^{+}}-y_{I_{1}^{+}}\right)+\left(y_{I_{0}}-y_{J_{0}}\right)<0$, thus, in the segment $\overline{A_{0} A_{1}^{+}}$, the successor function $f$ is monotonically decreasing, therefore, for system (2) there exists a unique point $S^{+} \in \overline{A_{0} A_{1}^{+}}$such that $f\left(B^{+}\right)=0$.

Next, in the region $\Omega_{1}$, the attractiveness of the periodic solution $\widehat{B^{+} B B^{+}}$is proved. See Figure 6(a), Let $g\left(G_{1}^{+}\right)=G_{2} \in M$, then $G_{2}$ jumps to point $G_{2}^{+} \in N$. In view of any two orbits

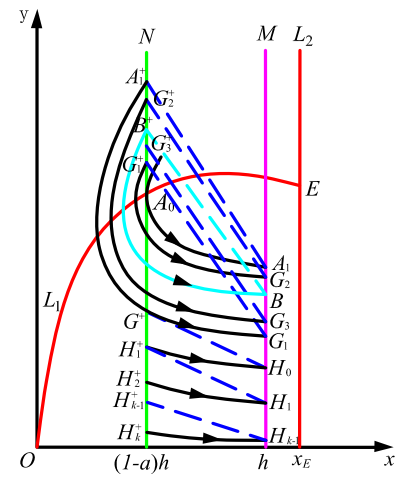

(a)

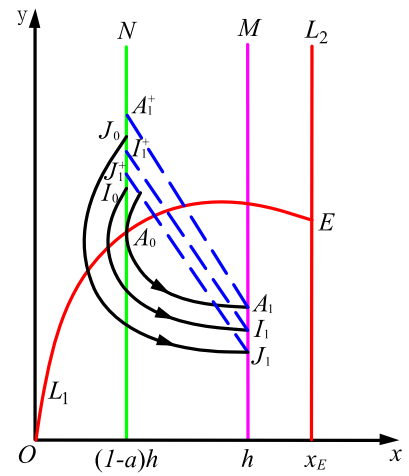

(b)

Figure 6 The schematic of the attractiveness of the order-1 periodic solution with conditions $0<(1-a) h<h \leq x_{E}<K$ and $y_{A_{0}}<y_{G_{1}^{+}}<y_{A_{1}^{+}}$. (a) The attractiveness of the order-1 periodic solution. (b) The uniqueness of the order-1 periodic solution 
are disjoint and $y_{A_{0}}<y_{G_{1}^{+}}<y_{A_{1}^{+}}$, then $y_{G_{1}}<y_{G_{2}}<y_{A_{1}}, y_{G_{1}^{+}}<y_{G_{2}^{+}}<y_{A_{1}^{+}}$. Let $g\left(G_{2}^{+}\right)=G_{3} \in M$, then $G_{3}$ jumps to point $G_{3}^{+} \in N$, we have $y_{G_{1}}<y_{G_{3}}<y_{G_{2}}, y_{G_{1}^{+}}<y_{G_{3}^{+}}<y_{G_{2}^{+}}$. Repeating the steps, we obtain two sequences $\left\{G_{k}\right\}_{k=1,2, \ldots} \in M$ and $\left\{G_{k}^{+}\right\}_{k=1,2, \ldots} \in N$ satisfying $y_{G_{1}^{+}}<\cdots<$ $y_{G_{2 k-1}^{+}}<y_{G_{2 k+1}^{+}}<\cdots<y_{G_{2 k}^{+}}<y_{G_{2 k-2}^{+}}<y_{G_{2}^{+}}$, then $f\left(G_{2 k-1}^{+}\right)=y_{G_{2 k}^{+}}-y_{G_{2 k-1}^{+}}>0$, and $f\left(G_{2 k}^{+}\right)=$ $y_{G_{2 k+1}^{+}}-y_{G_{2 k}^{+}}<0$. By the proof of Theorem 3.3, we get $\lim _{k \rightarrow+\infty} y_{G_{2 k-1}^{+}}=\lim _{k \rightarrow+\infty} y_{G_{2 k}^{+}}=y_{B^{+}}$.

The orbit with arbitrary point in segment $\overline{A_{1}^{+} G^{+}}$will intersect $N$ as time goes on, under the action of impulses, it passes through a point in segment $\overline{G_{2 k-1}^{+} G_{2 k+1}^{+}}$or $\overline{G_{2 k}^{+} G_{2 k-2}^{+}}$, here $G_{0}^{+}=A_{1}^{+}$. Similar to the discussion of Theorem 3.3, the orbit with arbitrary point in segment $\widehat{A_{1}^{+} G^{+}}$will be eventually attracted to the periodic solution $\widehat{B^{+} B B^{+}}$.

Assume a point $H_{0} \in M$ jumps to point $G^{+} \in N$ under the action of impulse. Let $\Pi\left(H_{0}\right)=$ $H_{1}^{+}$. Assume a point $H_{1} \in M$ jumps to point $H_{1}^{+} \in N$ under the action of impulse. Let $\Pi\left(H_{1}\right)=H_{2}^{+}$. Repeat the process until the phase set $N$ exists a $H_{K_{0}}^{+}\left(K_{0} \in Z_{+}\right)$such that $y_{H_{K_{0}}^{+}}<c$. Then there are two sequences $\left\{H_{k}\right\}_{k=1,2, \ldots K_{0}-1} \in M$ and $\left\{H_{k}^{+}\right\}_{k=1,2, \ldots K_{0}} \in N$ such that $\Pi\left(H_{k-1}\right)=H_{k}^{+}, y_{H_{k}^{+}}<y_{H_{k-1}^{+}}$, here $H_{0}^{+}=G^{+}$. For arbitrary point of $N$ below $G^{+}$, it must in segment $\overline{H_{k}^{+} H_{k+1}^{+}}$, where $k=1,2, \ldots, K_{0}$ and $y_{H_{k+1}^{+}}=0$. Under $k+1$ times the impulsive action, the orbit with arbitrary point that below $G^{+}$will passes through some point of segment $\overline{A_{1}^{+} G^{+}}$and will be attracted to the periodic solution $\widehat{B^{+} B B^{+}}$eventually. Thus, the order-1 periodic solution with the initial point that below $G^{+}$is nonexistent.

The orbit with arbitrary point above $A_{1}^{+}$of $N$ will intersect with $N$ at some point below $G^{+}$as time goes on, then the orbit will be attracted to the periodic solution $\widehat{B^{+} B B^{+}}$. Thus, the order-1 periodic solution with the initial point that above $A_{1}^{+}$is nonexistent.

Based on the above analysis, system (2) exists a unique order-1 periodic solution which is attractive. That completes the proof.

Like by the discussions of Theorem 3.3 and Theorem 3.4, we find the following.

Theorem 3.5 If $0<(1-a) h<x_{E}<h<K, x_{P_{2}} \geq(1-a) h$ and $y_{D^{+}} \leq y_{A_{0}}$, and system (2) exists a unique order-1 periodic solution, then the periodic solution in region $\Omega_{2}$ is attractive, where $\Omega_{2}=R_{+}-Q_{1}$ and $Q_{1}$ is an open region enclosed by orbit $\widehat{P_{0} P_{2} P_{1}}$ (see Figure $4(\mathrm{a})$ ). Meanwhile if $y_{D^{+}}>y_{A_{0}}$, then for system (2) there exists a unique order-1 periodic solution in region $\Omega_{2}$ which is attractive.

Theorem 3.6 If (ii) of II in Theorem 3.2 is true, and $y_{P_{1}^{+}} \leq y_{F_{2}}$ and system (2) exists a unique order-1 periodic solution, then the periodic solution in region $\Omega_{3}$ is attractive, where $\Omega_{3}=R_{+}-Q_{2}$ and $Q_{2}$ is an open region enclosed by orbit $\widehat{P_{0}} \widehat{F_{1} F_{2} P_{1}}$ (see Figure $4(\mathrm{~b})$ ). And if $y_{P_{1}^{+}}>y_{F_{2}}$, then for system (2) also there exists a unique order-1 periodic solution in region $\Omega_{3}$ which is attractive.

\subsection{Stability of order-1 periodic solution of system (2)}

Theorem 3.7 Denote $(\phi(t), \varphi(t))$ as a T-periodic solution in system (2), and $\phi_{0}=\phi(0)$, $\varphi_{0}=\varphi(0)$. Under the conditions of Theorem 3.2, if

$$
\left|\frac{\left[r(1-a) h\left(1-\frac{(1-a) h}{K}\right)-\alpha \sqrt{(1-a) h} \varphi_{0}\right]\left(\varphi_{0}-c\right)}{\left[r h\left(1-\frac{h}{K}\right)-\alpha \sqrt{h} \cdot \frac{\varphi_{0}-c}{1+b}\right](1-a) \varphi_{0}}\right|<1,
$$

then system (2) has a stable order-1 periodic solution. 
Proof It is easy to see that $\phi_{1}=\phi(T)=h, \varphi_{1}=\varphi(T) ; \phi_{1}^{+}=\phi\left(T^{+}\right), \varphi_{1}^{+}=\varphi\left(T^{+}\right)$, then

$$
\phi_{1}^{+}=\phi_{0}=(1-a) h, \quad \varphi_{1}^{+}=\varphi_{0}=(1+b) \varphi_{1}+c .
$$

Let $\Phi(x, y)=-a x, \Psi(x, y)=b y+c, \eta(x, y)=x-h$.

Then

$$
\begin{aligned}
\frac{\partial \Phi}{\partial x} & =-a, \quad \frac{\partial \Psi}{\partial y}=b, \quad \frac{\partial \eta}{\partial x}=1, \quad \frac{\partial \Psi}{\partial x}=\frac{\partial \Phi}{\partial y}=\frac{\partial \eta}{\partial y}=0, \\
\Delta_{1} & =\frac{P_{+}\left(\frac{\partial \Psi}{\partial y} \frac{\partial \eta}{\partial x}-\frac{\partial \Psi}{\partial x} \frac{\partial \eta}{\partial y}+\frac{\partial \eta}{\partial x}\right)+Q_{+}\left(\frac{\partial \Phi}{\partial x} \frac{\partial \eta}{\partial y}-\frac{\partial \Phi}{\partial y} \frac{\partial \eta}{\partial x}+\frac{\partial \eta}{\partial y}\right)}{P \frac{\partial \eta}{\partial x}+Q \frac{\partial \eta}{\partial y}} \\
& =\frac{P\left(\phi_{1}^{+}, \varphi_{1}^{+}\right)(b \times 1-0 \times 0+1)+Q\left(\phi_{1}^{+}, \varphi_{1}^{+}\right)(-a \times 0+0 \times 1+0)}{P\left(\phi_{1}, \varphi_{1}\right) \times 1+Q\left(\phi_{1}, \varphi_{1}\right) \times 0} \\
& =\frac{r \phi_{0}\left(1-\frac{\phi_{0}}{K}\right)-\alpha \sqrt{\phi_{0}} \varphi_{0}}{r \phi_{1}\left(1-\frac{\phi_{1}}{K}\right)-\alpha \sqrt{\phi_{1}} \varphi_{1}},
\end{aligned}
$$

and

$$
\begin{aligned}
\int_{0}^{T}\left(\frac{\partial P}{\partial x}+\frac{\partial Q}{\partial y}\right) d t & =\int_{0}^{T}\left(r-\frac{2 r}{K} x(t)-\frac{\alpha y(t)}{2 \sqrt{x(t)}}-d+\alpha \beta \sqrt{x(t)}\right) d t \\
& =\int_{0}^{T}\left(\frac{\dot{\phi(t)}}{\phi(t)}+\frac{\dot{\varphi(t)}}{\varphi(t)}\right) d t \\
& =\int_{0}^{T} d \ln \phi(t) \varphi(t) \\
& =\ln \frac{\phi(1) \varphi(1)}{\phi(0) \varphi(0)} .
\end{aligned}
$$

Thus

$$
\begin{aligned}
\mu_{2} & =\Delta_{1} \exp \int_{0}^{T}\left(\frac{\partial P}{\partial x}+\frac{\partial Q}{\partial y}\right) d t \\
& =\frac{r \phi_{0}\left(1-\frac{\phi_{0}}{K}\right)-\alpha \sqrt{\phi_{0}} \varphi_{0}}{r \phi_{1}\left(1-\frac{\phi_{1}}{K}\right)-\alpha \sqrt{\phi_{1}} \varphi_{1}} \times \frac{\phi(1) \varphi(1)}{\phi(0) \varphi(0)} \\
& =\frac{\left[r(1-a) h\left(1-\frac{(1-a) h}{K}\right)-\alpha \sqrt{(1-a) h} \varphi_{0}\right]\left(\varphi_{0}-c\right)}{\left[r h\left(1-\frac{h}{K}\right)-\alpha \sqrt{h} \cdot \frac{\varphi_{0}-c}{1+b}\right](1-a) \varphi_{0}} .
\end{aligned}
$$

Therefore, $\left|\mu_{2}\right|<1$, and by Lemma 2.2, system (2) has a stable order-1 periodic solution.

\section{Simulations and conclusion}

We show an example in this section. For system (2), let $r=1.2, K=1.8, \alpha=0.85, \beta=0.7$, $d=0.5$. By calculation, the equilibrium point of system (4) is $E(0.7062,0.7209)$. Then we 


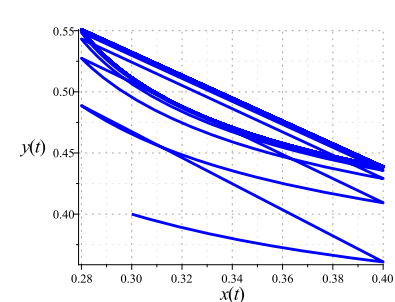

(a)

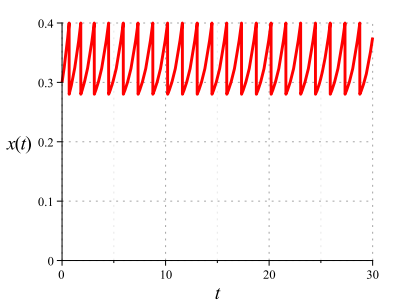

(b)

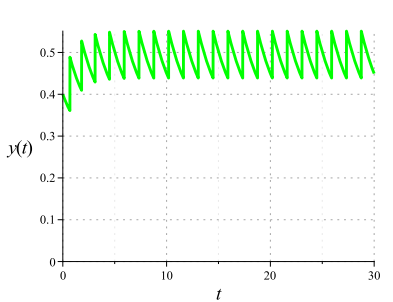

(c)

Figure 7 Numerical simulations in Case I. (a) Phase portrait of $x(t)$ and $y(t)$ on $h=0.4$. (b) Time series of $x(t)$. (c) Time series of $y(t)$

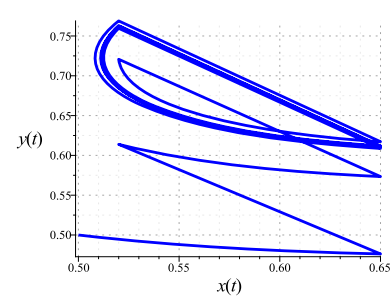

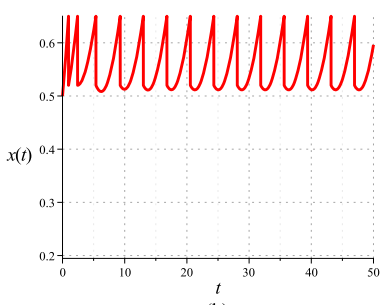

(b)

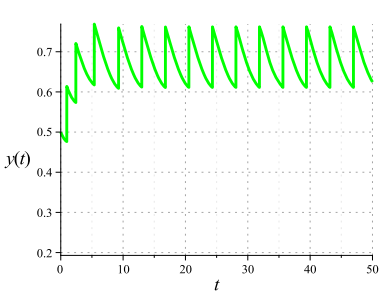

(c)

Figure 8 Numerical simulations in Case I. (a) Phase portrait of $x(t)$ and $y(t)$ on $h=0.65$. (b) Time series of $x(t)$. (c) Time series of $y(t)$

have

$$
\left\{\begin{array}{l}
x^{\prime}(t)=1.2 x(t)\left(1-\frac{x(t)}{1.8}\right)-0.85 \sqrt{x(t)} y(t), \\
y^{\prime}(t)=y(t)(0.595 \sqrt{x(t)}-0.5), \\
\left.\begin{array}{l}
\Delta x(t)=-a x(t), \\
\Delta y(t)=b y(t)+c,
\end{array}\right\} \quad x=h .
\end{array}\right\}
$$

Let $h=0.4, a=0.3, b=-0.2$ and $c=0.2$ satisfy the condition $0<(1-a) h<h<x_{E}<K$, and the initial value is $(0.3,0.4)$ satisfying $x_{0}<h$. Figure 7 indicates that for system (6) there exists an order-1 periodic solution which is stable, and arbitrary orbit of system (6) is attracted to the periodic solution.

Let $h=0.65, a=0.2, b=0.1$ and $c=0.09$ satisfy the condition $0<(1-a) h<h<x_{E}<K$ and the initial value be $(0.5,0.5)$. Figure 8 illustrates that for system $(6)$ there exists a stable order-1 periodic solution, and an arbitrary orbit of system (6) is attracted to the periodic solution.

The phase portrait and time series of prey density and predator density with condition $0<(1-a) h<x_{E}<h<K$ are shown in Figure 9 for $h=0.8, a=0.4, b=0.15$ and $c=0.1$ with the initial value $(0.5,0.6)$. It indicates that for system $(6)$ there exists a stable order-1 periodic solution, and an arbitrary orbit of system (6) is attracted to the periodic solution.

In order to prevent the extinction of predator under the herd behavior of the prey (such as the drifting herbivores observed in the savanna), this paper presents a prey-predator system with square root response function under state-dependent control strategy. In different cases, we discuss the existence of the order-1 periodic solution by the successor function method. Then we analyze the uniqueness and attractiveness of the periodic so- 

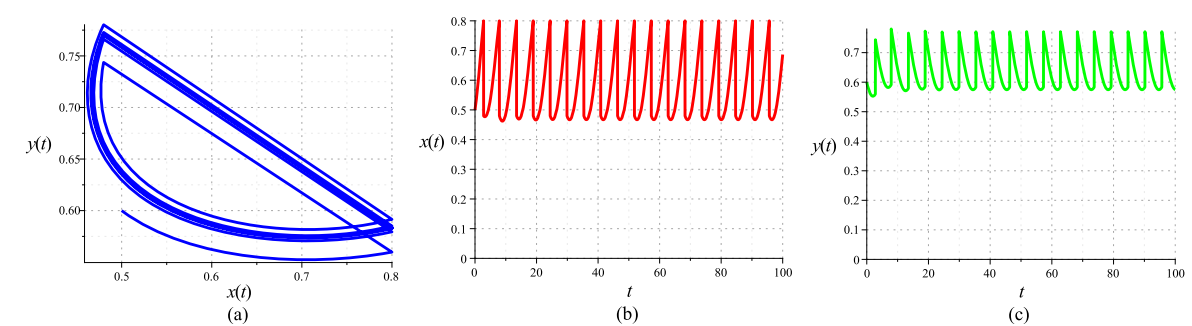

Figure 9 Numerical simulations in Case II. (a) Phase portrait of $x(t)$ and $y(t)$ on $h=0.8$. (b) Time series of $x(t)$. (c) Time series of $y(t)$

lution. Furthermore, we prove order-1 periodic solution is stable under certain conditions. Numerical simulations with an example are carried out which illustrate that the state-dependent impulse control strategy is effective. Compared with the literature [16], our research is more comprehensive, which is an improvement and complement for the results of the above literature.

Acknowledgements

The paper was supported by supported by the National Natural Science Foundation of China (No. 11371230).

\section{Competing interests}

The authors declare that they have no competing interests.

Authors' contributions

All authors read and approved the final manuscript.

\section{Publisher's Note}

Springer Nature remains neutral with regard to jurisdictional claims in published maps and institutional affiliations.

Received: 23 October 2017 Accepted: 27 January 2018 Published online: 20 February 2018

\section{References}

1. Braza, P.A.: Predator-prey dynamics with square root functional responses. Nonlinear Anal., Real World Appl. 13(4), 1837-1843 (2012)

2. Sk, B., Alam, S.: A dynamics of a prey-predator model with defence mechanism for prey. Int. J. Adv. Appl. Math. Mech. 4(3), 55-59 (2017)

3. Matia, S.N., Alam, S.: Prey-predator dynamics under herd behavior of prey. Univers. J. Appl. Math. 1(4), $251-257$ (2013)

4. Gimmelli, G., Kooi, B.W., Venturino, E.: Ecoepidemic models with prey group defense and feeding saturation. Ecol. Complex. 22, 50-58 (2015)

5. Banerjee, M., Kooi, B.W., Venturino, E.: An ecoepidemic model with prey herd behavior and predator feeding saturation response on both healthy and diseased prey. Math. Model. Nat. Phenom. 12(2), 133-161 (2017)

6. Cheng, H., Wang, F., Zhang, T.: Multi-state dependent impulsive control for Holling I predator-prey model. Discrete Dyn. Nat. Soc. 2012(12), 30-44 (2012)

7. Sun, K., Zhang, T., Tian, Y.: Dynamics analysis and control optimization of a pest management predator-prey model with an integrated control strategy. Appl. Math. Comput. 292, 253-271 (2017)

8. Zhang, S., Meng, X., Feng, T., Zhang, T.: Dynamics analysis and numerical simulations of a stochastic non-autonomous predator-prey system with impulsive effects. Nonlinear Anal. Hybrid Syst. 26, 19-37 (2017)

9. Cheng, H., Wang, F., Zhang, T.: Multi-state dependent impulsive control for pest management. J. Appl. Math. 2012 Article ID 381503 (2012)

10. Meng, X., Zhang, L.: Evolutionary dynamics in a Lotka-Volterra competition model with impulsive periodic disturbance. Math. Methods Appl. Sci. 39(2), 177-188 (2016)

11. Zhang, T., Ma, W., Meng, X.: Global dynamics of a delayed chemostat model with harvest by impulsive flocculant input. Adv. Differ. Equ. 2017(1), 115 (2017)

12. Liu, Q., Huang, L., Chen, L.: A pest management model with state feedback control. Adv. Differ. Equ. 2016(1), 292 (2016)

13. Tian, Y., Zhang, T., Sun, K.: Dynamics analysis of a pest management prey-predator model by means of interval state monitoring and control. Nonlinear Anal. Hybrid Syst. 23, 122-141 (2017)

14. Liu, L., Meng, X.: Optimal harvesting control and dynamics of two-species stochastic model with delays. Adv. Differ. Equ. 2017(1), 18 (2017)

15. Wang, J., Cheng, H., Meng, X., Pradeep, B.S.A.: Geometrical analysis and control optimization of a predator-prey model with multi state-dependent impulse. Adv. Differ. Equ. 2017(1), 252 (2017) 
16. Sun, S., Guo, C., Qin, C.: Dynamic behaviors of a modified predator-prey model with state-dependent impulsive effects. Adv. Differ. Equ. 2016(1), 50 (2016)

17. Chen, L.: Pest control and geometric theory of semi-continuous dynamical system. J. Beihua Univ. Nat. Sci. 12(1), 1-9 (2011)

18. Zhang, T., Ma, W., Meng, X., Zhang, T.: Periodic solution of a prey-predator model with nonlinear state feedback control. Appl. Math. Comput. 266, 95-107 (2015)

19. Liu, B., Tian, Y., Kang, B.: Dynamics on a Holling II predator-prey model with state-dependent impulsive control. Int. J. Biomath. 5(3), 675 (2012)

20. Zhang, T., Meng, X., Liu, R., Zhang, T.: Periodic solution of a pest management Gompertz model with impulsive state feedback control. Nonlinear Dyn. 78(2), 921-938 (2014)

21. Zhao, W., Liu, Y., Zhang, T., Meng, X.: Geometric analysis of an integrated pest management model including two state impulses. Abstr. Appl. Anal. 2014(1), 91506 (2014)

22. Zhang, T., Zhang, J., Meng, X., Zhang, T.: Geometric analysis of a pest management model with Holling's type III functional response and nonlinear state feedback control. Nonlinear Dyn. 84(3), 1529-1539 (2016)

23. Cheng, H., Zhang, T., Wang, F.: Existence and attractiveness of order one periodic solution of a Holling I predator-prey model. Abstr. Appl. Anal. 2012, Article ID 126018 (2012)

\section{Submit your manuscript to a SpringerOpen ${ }^{\circ}$ journal and benefit from:}

- Convenient online submission

- Rigorous peer review

- Open access: articles freely available online

- High visibility within the field

Retaining the copyright to your article

Submit your next manuscript at $\boldsymbol{~ s p r i n g e r o p e n . c o m ~}$ 